\title{
Inhibitory connections in the honeybee antennal lobe are spatially patchy
}

\author{
Cyrille C. Girardin, Sabine Kreissl, and C. Giovanni Galizia \\ Department of Neurobiology, University of Konstanz, Konstanz, Germany
}

\begin{abstract}
Girardin CC, Kreissl S, Galizia CG. Inhibitory connections in the honeybee antennal lobe are spatially patchy. J Neurophysiol 109: 332-343, 2013. First published October 24, 2012; doi:10.1152/jn.01085 2011.-The olfactory system is a classical model for studying sensory processing. The first olfactory brain center [the olfactory bulb of vertebrates and the antennal lobe (AL) of insects] contains spherical neuropiles called glomeruli. Each glomerulus receives the information from one olfactory receptor type. Interglomerular computation is accomplished by lateral connectivity via interneurons. However, the spatial and functional organization of these lateral connections is not completely understood. Here we studied the spatial logic in the AL of the honeybee. We combined topical application of neurotransmitters, olfactory stimulations, and in vivo calcium imaging to visualize the arrangement of lateral connections. Suppression of activity in a single glomerulus with $\gamma$-aminobutyric acid (GABA) while presenting an odor reveals the existence of inhibitory interactions. Stimulating a glomerulus with acetylcholine (ACh) activates inhibitory interglomerular connections that can reduce odor-evoked responses. We show that this lateral network is patchy, in that individual glomeruli inhibit other glomeruli with graded strength, but in a spatially discontinuous manner. These results suggest that processing of olfactory information requires combinatorial activity patterns with complex topologies across the AL.
\end{abstract}

acetylcholine; antennal lobe; GABA; honeybee; iontophoresis

IN SENSORY SYSTEMS, information from the receptor cells are processed via networks of neurons that interconnect laterally. This primary transformation modifies the temporal and spatial representation of the stimulus. Studying the logic of lateral interconnectivity in early sensory processing is therefore important to understanding how the brain creates reliable representations of the environment. In the olfactory system, these lateral interactions occur first in the antennal lobe (AL) (insects) or in the olfactory bulb (vertebrates).

Axons of olfactory receptor cells and the processes of olfactory bulb or of antennal lobe neurons form glomeruli. The excitatory transmitter of receptor cells in insects is likely to be acetylcholine $(\mathrm{ACh})$. In the honeybee, 160 glomeruli are arranged on the surface of the AL, creating a spherical twodimensional layer. Several populations of local neurons interconnect glomeruli: homogeneous local neurons branch in large areas of the AL in a uniform manner, while heterogeneous local neurons innervate only one glomerulus densely and others sparsely, allowing for more selective interglomerular connections (Flanagan and Mercer 1989; Fonta et al. 1993; Sun et al. 1993). A large subpopulation of $\sim 800$ local neurons is GABAergic (Schäfer and Bicker 1986) and creates a local inhibitory network. Physiological experiments revealed excitatory and inhibitory lateral interactions in the AL of insects and

Address for reprint requests and other correspondence: C. C. Girardin, Dept. of Neurobiology, University of Konstanz, Universiteatstrasse 10, 78457 Konstanz, Germany (e-mail: cyrille.girardin@uni-konstanz.de). in the olfactory bulb of vertebrates (Aungst et al. 2003; Christensen et al. 1998a, 1998b; Luo and Katz 2001; Olsen et al. 2007; Olsen and Wilson 2008; Reisenman et al. 2008; Root et al. 2007; Shang et al. 2007; Vucinic et al. 2006; Yokoi et al. 1995).

In vertebrates, geometrical arrangements (center-surround), patchy arrangements (possibly dictated by functional relationships), and intermediate arrangements have been proposed, leaving the question unresolved at the current stage (Fantana et al. 2008; Kim et al. 2011, 2012; Luo and Katz 2001). Here we specifically studied the spatial organization of lateral inhibitory connections. In particular, we asked whether the strength of inhibitory connections depends on distance, and whether activity in a single glomerulus can alter the spatial response pattern driven by an odor. Both $\gamma$-aminobutyric acid (GABA) and $\mathrm{ACh}$ are present in the bee AL, as shown in anatomical studies (Kreissl and Bicker 1989; Schäfer and Bicker 1986). Functionally, GABA and ACh suppress and increase neuronal activity, respectively (Michelsen and Braun 1987; Waldrop et al. 1987; Waldrop and Hildebrand 1989). Data from different species support the idea that GABA and ACh act on most or possibly all local neurons and projection neurons (see Chou et al. 2010; Christensen et al. 1998a; Distler and Boeckh 1998; Wilson 2011). Thus we used GABA and ACh to manipulate the activity in single glomeruli. We show that there is no correlation between distance and inhibitory strength between a glomerulus pair. Furthermore, activating a single glomerulus can modulate the spatial odor response pattern.

\section{MATERIALS AND METHODS}

Animal preparation and staining with fura. A total of 36 adult forager honeybees (female) were used for calcium imaging of olfactory projection neurons in the AL with fura- 2 . The detailed procedures have been described elsewhere (Fernandez et al. 2009; Sachse and Galizia 2002; Szyszka et al. 2005). Briefly, adult forager honeybees were collected at the hive entrance. They were immobilized by cooling on ice and fixed in a Plexiglas recording chamber with wax. The head capsule was opened. Trachea and glands covering the staining location were removed. A glass electrode coated with fura-2 dextran was inserted bilaterally between the calyces of the mushroom bodies to stain the projection neurons retrogradely. The capsule was closed again and sealed with wax. Bees were fed to satiation with sugar water $(30 \%)$ and kept overnight in a humidified box at room temperature. The next day, the animal was prepared for optical recording. Antennae were fixed with wax. To minimize movement artifact the mandibles and the proboscis were fixed with wax. The esophagus was stretched, and the abdomen was slightly compressed with a sponge. The head capsule was reopened. Glands and trachea covering $\mathrm{AL}$ were removed.

Odor stimulation and iontophoresis. A custom-built olfactometer using magnetic two-way valves was used for odor stimulation. Three odors (1-nonanol, 1-hexanol, and 2-heptanone, diluted 1:100 in mineral oil) and a mineral oil control were used. This concentration was 
appropriate to allow fast return (2-10 s depending on the glomerulus, see Supplemental Movie S3) ${ }^{1}$ to baseline after termination of the odor pulse. These odors were presented (1-3 s) at the beginning of each experiment to test odor response and to identify glomeruli with the atlas (Sachse et al. 1999). The same odors were also presented in combination with neurotransmitter injection.

Drugs were dissolved in saline. Fresh solutions were prepared every week. Multibarrel pipettes were used to inject $\mathrm{ACh}(0.5 \mathrm{M}, \mathrm{pH}$ 4.2) and GABA (0.5 M, pH 3.5) with iontophoresis (Axoclamp 2A, Axon Instruments or MVSC-02C, npi electronic). One barrel (filled with saline, $\mathrm{pH}$ 7.6) was used as current balance channel. Injection current ranged from +10 to $+100 \mathrm{nA}$ for both GABA and ACh. For $\mathrm{ACh}$ injections the current was set to produce responses that were within the range of odor responses (see Supplemental Movie S3). Higher currents (up to 1,000 nA) were only used for current tests and $\mathrm{pH}$ tests. A retention current $(-5 \mathrm{nA})$ was applied between injections. Injections lasted 1-4 s. Each drug barrel was tested, and the pipette was only used if effects (excitation with ACh and odor response suppression with GABA) were visible at the injection site. For each bee we first recorded the calcium response pattern to the odor 1-nonanol, and then one of the strongly responding glomeruli (T1-17 and T1-33; see Sachse et al. 1999) was penetrated with the pipette.

Since in some experiments no balancing current was used, we tested the effects of current in control experiments (see Fig. 2). We used three-barreled pipettes for current tests. One barrel was filled with ACh (0.5 M, pH 4.2), and the two remaining barrels were filled with saline ( $\mathrm{pH}$ 7.6). We used one of the saline barrels to balance the current. The other was used to inject saline and thus test directly the effect of current. We tested different injection currents for saline and $\mathrm{ACh}$ with the current balance module switched ON or OFF. For $\mathrm{pH}$ tests three-barrel pipettes were used and filled with ACh $(0.5 \mathrm{M}$, $\mathrm{pH} 4.2$ ), saline with $\mathrm{pH} 7.6$ (current balance channel), and saline with pH 3.7 (injected to test $\mathrm{pH}$ ). Different injection currents were tested for $\mathrm{ACh}$ and saline with $\mathrm{pH} 3.7$, and current was either balanced or not with the barrel with saline of $\mathrm{pH} 7.6$ (see Fig. 2).

Imaging and data analysis. We used ratiometric calcium imaging (dip objective $\times 20,0.5 \mathrm{NA}$, Olympus; excitation wavelengths 340 and $380 \mathrm{~nm}$ and emission $510 \mathrm{~nm}$ ). Data were acquired at $5 \mathrm{~Hz}$ with a CCD camera (Till Photonics). Using a CCD camera allowed us to image a large portion of the $\mathrm{AL}$ at high spatial resolution (image: $172 \times 130$ pixels corresponding to $430 \times 325 \mu \mathrm{m}$ ). Unlike confocal or two-photon systems, a CCD camera records the whole field of view simultaneously because no scanning time is needed.

Data were analyzed with custom-written routines in IDL (Research Systems, Boulder, CO) and MATLAB (MathWorks). Lateral movement correction was performed by manually realigning each measurement within one animal. Ratios of $340 \mathrm{~nm}$ to $380 \mathrm{~nm}$ were calculated, and offset was removed by subtracting the mean signal before injection or odor stimulation. Glomeruli were selected based on morphological pictures and on activity in response to odor stimulation. Time traces were calculated for the selected glomeruli. Quantitative analyses were performed on time traces; false-color pictures only served for display purposes and glomerulus identification by comparing with the atlas (Sachse et al. 1999). False-color pictures in Figs. 1 and 3 were calculated by subtracting the background response ( 3 frames averaged) from the response during odor stimulation or injection ( 3 frames averaged). The false-color picture in Fig. 5 was calculated by subtracting the mean background response (18 trials, 35 frames averaged) from the response (18 trials, 2 frames averaged) just after injection termination. The picture was then spatially filtered for display purpose. An AL was considered to have inhibitory response due to ACh injection if at least one glomerulus showed a clear negative deflection in the signal (24/36 ALs, always only 1 AL per bee). These ALs were analyzed further (in the other ALs excitatory responses were detected

${ }^{1}$ Supplemental Material for this article is available online at the Journal website. at the injection site, but no clear negative signal was visible in any other glomerulus). We did not investigate the reason why 12 ALs showed no inhibition. Possible reasons include too small injection volumes, damage to the tissue by the pipette, or a very sparse inhibitory network so that all inhibited glomeruli were outside the field of view. The responses of single glomeruli were calculated by averaging $5 \mathrm{~s}$ starting at injection time. For experiments with multiple injections using the same current the mean response was calculated. For statistical analysis see below. To compare the results from several bees (see Fig. $6 B$ ) we pooled data and normalized responses (maximum inhibition set to -1 and other responses linearly rescaled). In experiments with combined odor and ACh injections (see Fig. 9) the odor response suppression was estimated by subtracting the response to odor + injection from the response to odor alone. Odor response was taken as the mean during odor presentation ( $1 \mathrm{~s})$ and the inhibition as the integrated response over $5 \mathrm{~s}$ from injection time. Data are presented as means $\pm \mathrm{SE}$ unless otherwise stated.

We measured the radius of activity produced by ACh injection on the ratiometric data $\left(\Delta \mathrm{F}_{340} / \mathrm{F}_{380}\right)$. The response of each pixel was calculated by averaging $1 \mathrm{~s}$ of activity during the injection and by spatial averaging with $11 \times 11$ pixels $(27.5 \times 27.5 \mu \mathrm{m})$. We plotted the response to the injection for all pixels along a line passing through the center of the injection site (ACh response profile of the injection site) and similarly for the response to an odor at the same location (odor response profile at the injection site). We then fitted a Gaussian equation to each response profile and measured the half-width at half-height on the fits to compare the radius of activity produced by $\mathrm{ACh}$ and odors at the injection site.

Statistical analysis. Unless otherwise mentioned, significance was determined with two-sample $t$-tests (2-tailed). For multiple comparisons the significance level was adjusted with the Bonferroni-Holm correction.

Anatomical analysis. Biocytin (2\%) was injected iontophoretically $(+1,000 \mathrm{nA}, 1 \mathrm{~s}$ ON/OFF cycles, $10 \mathrm{~min})$ extracellularly into a glomerulus after recording. Dissection, visualization of biocytin, and tissue processing were according to standard procedures as described in Kreissl et al. (2010). Briefly, brains were dissected in cold 4\% paraformaldehyde and fixed for $2 \mathrm{~h}$ before being washed extensively in phosphate-buffered saline (PBS). DAPI was used as a counterstain, and biocytin was tagged with streptavidin-Cy3 (Jackson Immunoresearch Europe) at 1:500 in PBS for at least 3 days at room temperature. Brains were washed, dehydrated, and cleared in xylene, and embedded in DPX (Sigma-Aldrich Chemie, München, Germany). Brains were scanned with a Zeiss LSM 510 NL confocal microscope (Carl Zeiss, Jena, Germany). Stained cells were traced and reconstructed with Amira (Visage Imaging, Berlin, Germany).

\section{RESULTS}

Acetylcholine produces excitatory responses. We injected $\mathrm{ACh}$ in AL glomeruli of bees with a pipette by iontophoresis. ACh produced strong intracellular increases of calcium concentration at the injection site (Fig. 1). Typically, the response to ACh started one or two sampling frames (200-400 ms) after injection onset, when the current was between +30 and $+80 \mathrm{nA}$. With these currents the response peaked $0.75 \pm 0.24 \mathrm{~s}$ (mean \pm SE, $n=13$ bees) after injection offset. In general, the calcium level returned to baseline in $<30 \mathrm{~s}$ even with relatively high currents (Fig. 2). The signal amplitude increased with increasing injection current (Fig. 1B). The excitatory effect was local and did not affect the entire AL directly. The radius of activity produced by ACh injections $(30.6 \pm 3.1 \mu \mathrm{m})$ was not significantly different from that produced by an odor stimulation (29.7 $\pm 2.0 \mu \mathrm{m}$, mean $\pm \mathrm{SE}, n=9$ bees; paired $t$-test, 2 -tailed, $\left.t_{16}=0.31, P=0.81\right)$. Cell bodies at the periphery of the AL 
Fig. 1. Response of glomeruli and cell bodies to acetylcholine ( $\mathrm{ACh}$ ) injection. $A$, top: example of raw picture of a right antennal lobe (AL) taken with an incident light wavelength of $380 \mathrm{~nm}$ (sum of all frames in this recording). Bottom: corresponding false-color picture of response (color bar on left) to a single $\mathrm{ACh}$ injection $(+80 \mathrm{nA}, 1.6 \mathrm{~s})$. Note the responding cell body (black arrowhead; other cell bodies of this glomerulus are likely out of focus). Red arrow points to the injected glomerulus. an, Antennal nerve, 1 , lateral. Scale bar, $100 \mu \mathrm{m}$ (applies to both panels). False-color pictures calculated by subtracting the background response ( 3 frames averaged before stimulus) from the response during odor stimulation ( 3 frames averaged just after odor) $(\Delta \mathrm{F}) . B$ : time traces of the responses to injections of $\mathrm{ACh}$ (gray bar) in the glomerulus shown in $A$. The responses of the glomerulus (red) and of a cell body (black) are shown for $+30 \mathrm{nA}$ (top) and $+80 \mathrm{nA}$ (bottom). Time traces represent the average of $11 \times 11$ pixels $(27.5 \mu \mathrm{m} \times$ $27.5 \mu \mathrm{m}$ ) around the glomerulus or the cell body center.

also responded during $\mathrm{ACh}$ stimulation with a similar time course (Fig. 1B) and a short delay (close to our temporal resolution of $200 \mathrm{~ms}$ ). We assume that these are the cell bodies of projection neurons in the injected glomerulus, suggesting that $\mathrm{ACh}$ depolarized the measured projection neurons, and that these cells express voltage-sensitive calcium channels on their somata. Therefore, $\mathrm{ACh}$ injections can be used to generate localized activity spots (Fig. $1 A$ ).

Next, we verified that the effect was due to $\mathrm{ACh}$ and not to the injecting current or the low $\mathrm{pH}$ of the solution by injecting
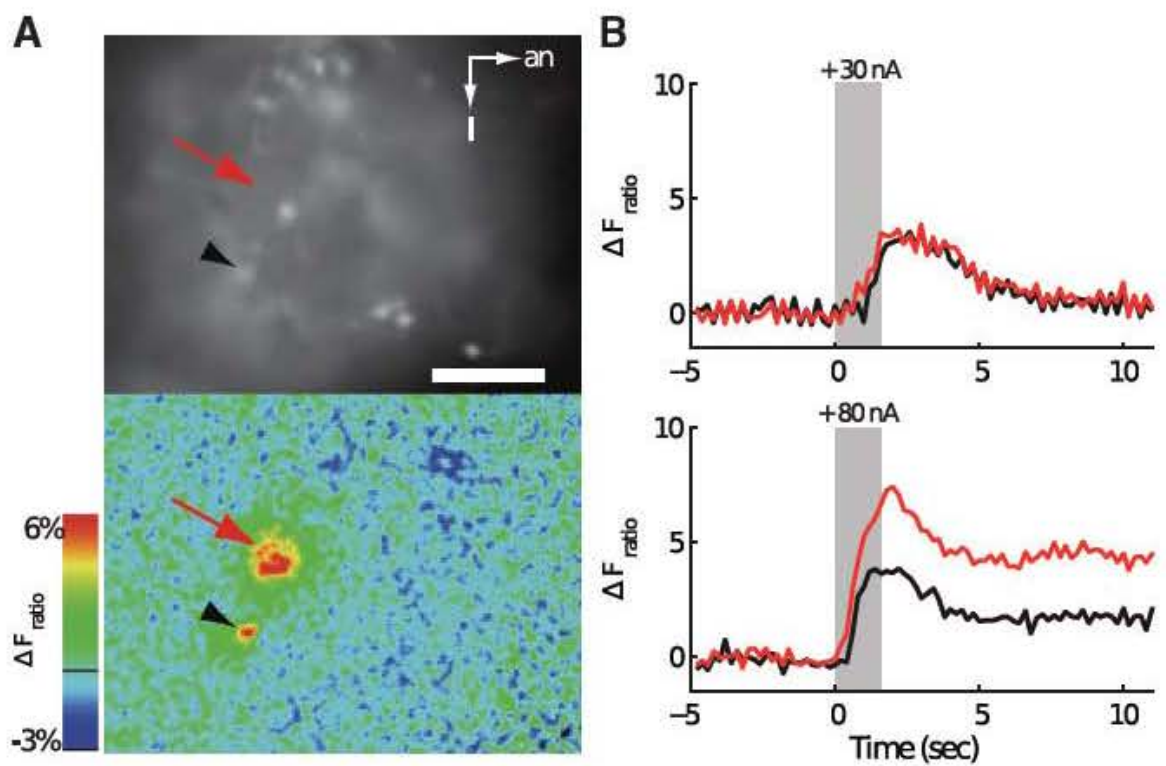

saline of $\mathrm{pH} 7.6$ (current test) and $\mathrm{pH} 3.7$ (pH test). For comparison we also injected $\mathrm{ACh}$ at the same locations (Fig. 2 ). In all cases $\mathrm{ACh}$ produced an excitatory response at the injection site (Fig. 2A). In two of three bees injections of saline $\mathrm{pH} 7.6$ with currents up to $+1,000 \mathrm{nA}$ did not produce any measurable response in the injected glomerulus. In the third bee a small excitatory response was visible with $+160 \mathrm{nA}$ (Fig. $2 B$ ). However, this response was of negligible amplitude compared with the response triggered by $\mathrm{ACh}$ with the same current (Fig. 2A). Moreover, ACh produced an inhibitory
Fig. 2. Effect of saline and current injections. In $A-F$ the gray bar represents injection time. $A$ : responses to $\mathrm{ACh}$ at the injection location. Current was $+160 \mathrm{nA}$ in all traces. Black, 2 examples with current balanced; blue, current not balanced. $B$ : responses to saline $(\mathrm{pH} \mathrm{7.6)}$ at the injection location shown in $A$. Current was $+160 \mathrm{nA}$ in all traces. Black, 2 examples with current balanced; blue, current not balanced. Saline only triggered a very small excitatory signal. Note the scale difference in $A$ and $B . C$ : response of a distant glomerulus to the injections of $\mathrm{ACh}$ shown in $A$. Lateral inhibition was clearly visible in this glomerulus (see text for details). $D$ : response of a distant glomerulus to the injections of saline shown in $B$. The small effect of saline at the injection location $(B)$ was not large enough to trigger lateral inhibition in the distant glomerulus, while $\mathrm{ACh}$ did produce lateral inhibition $(C) . E$ : responses to $\mathrm{ACh}$ at the injection location in a different bee. Current was balanced and was $+80 \mathrm{nA}$ (red), $+100 \mathrm{nA}$ (green), $+150 \mathrm{nA}$ (black, repetition in blue). $F$ : responses to saline $(\mathrm{pH} 3.7)$ at the injection location shown in $E$. Current was $+50 \mathrm{nA}$, not balanced (red), $+300 \mathrm{nA}$, not balanced (green), $+1,000 \mathrm{nA}$, not balanced (blue), $+1,000 \mathrm{nA}$, balanced (black). Only very high and unbalanced current (blue) could trigger excitation at the injection location.
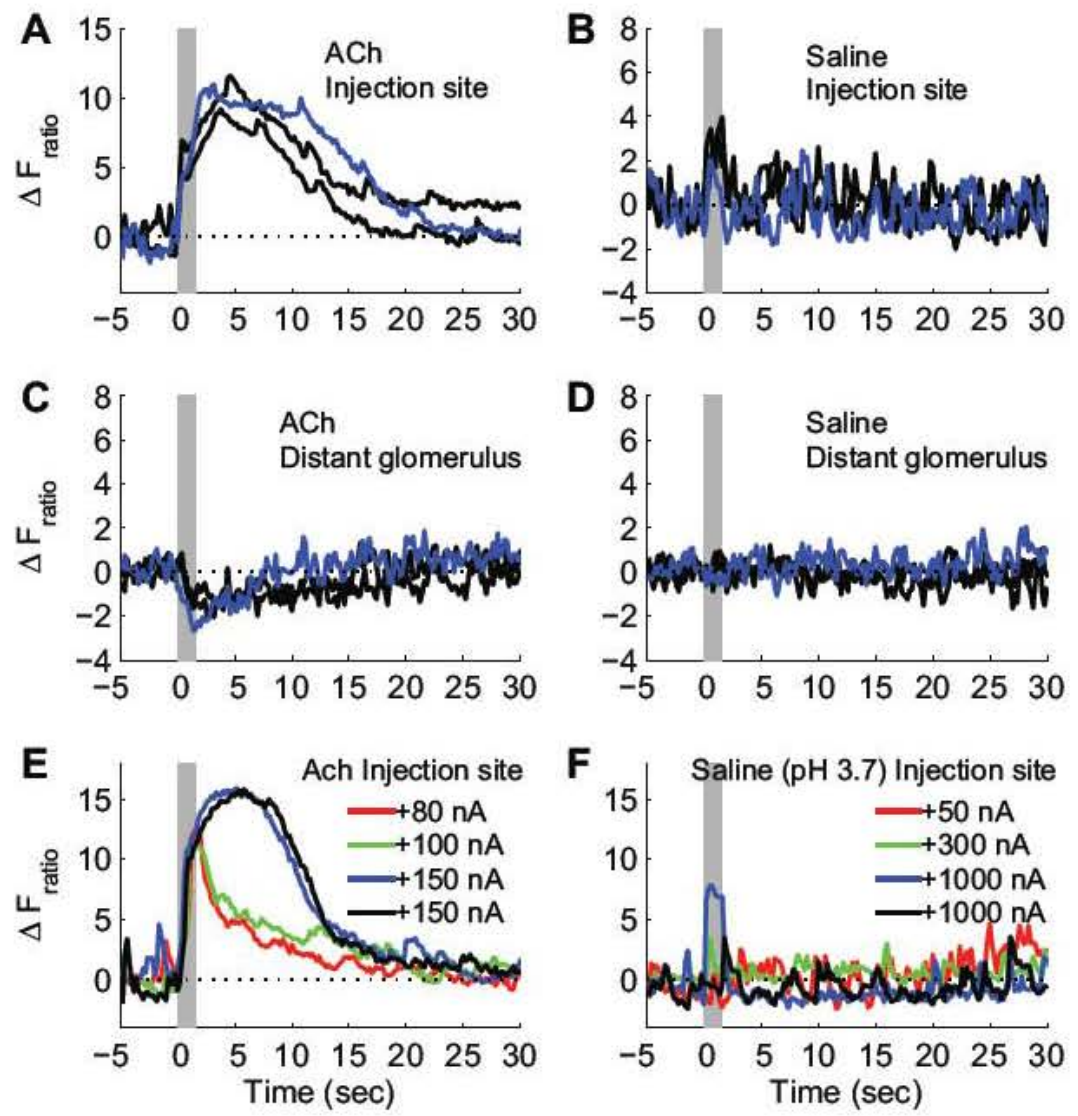
response in other, distant glomeruli (Fig. 2C; see below), while saline did not (Fig. 2D). Balancing or not balancing the current (see MATERIALS AND METHODS) had no effect.

To assess the effect of the low $\mathrm{pH}$ in the $\mathrm{ACh}$ and GABA solutions we injected saline with $\mathrm{pH} 3.7$ or $\mathrm{ACh}$ at the same locations. Typically saline with low $\mathrm{pH}$ could only produce a response with a current equal to or larger than $+300 \mathrm{nA}$ and when current was not balanced (Fig. 2, $E$ and $F$ ). Note that in the current and $\mathrm{pH}$ test experiments we used particularly high currents (up to $1,000 \mathrm{nA}$ ) compared with standard conditions $(10-100 \mathrm{nA})$. This was to maximize potential current or $\mathrm{pH}$ effects. We conclude that current and $\mathrm{pH}$ have no influence under our standard protocol (injection current $\leq+100 \mathrm{nA}$ ). We performed additional controls with high currents and long injection times to test diffusion of $\mathrm{ACh}$. We also observed that diffusion of ACh away from the injection location was slow and was only effective with large currents $(>+200 \mathrm{nA})$. For example, an ACh injection with $+1,000 \mathrm{nA}(1.6 \mathrm{~s})$ triggered an excitatory response at $125 \mu \mathrm{m}$ from the pipette after $\sim 15 \mathrm{~s}$. No lateral diffusion effects were visible with the parameters used in the remainder of this report.

$G A B A$ suppresses odor response. After showing that the excitatory neurotransmitter $\mathrm{ACh}$ elicits neural activity, we asked whether the inhibitory transmitter GABA would block activity, given that many local neurons in the AL are GABAergic (Schäfer and Bicker 1986). To this end, we elicited glomerular activity with the odor 1-nonanol, which activates glomeruli T1-17 and T1-33 (Sachse et al. 1999). We then targeted one of these two glomeruli with a multibarrel pipette containing GABA. Next, we combined GABA injections with odor stimulation. We found that GABA injections reduced odor responses in the injected glomerulus ( $n=7$ bees; see Supplemental Movie S2) and that the strength of suppression was dose dependent both for injection duration and for current intensity (data not shown). As for $\mathrm{ACh}$ injections, the direct effect of GABA injections was restricted to the targeted glomerulus (Fig. 3, $A-C$ ). For example, GABA injection into T1-17 did not influence the response of the direct neighbor T1-33
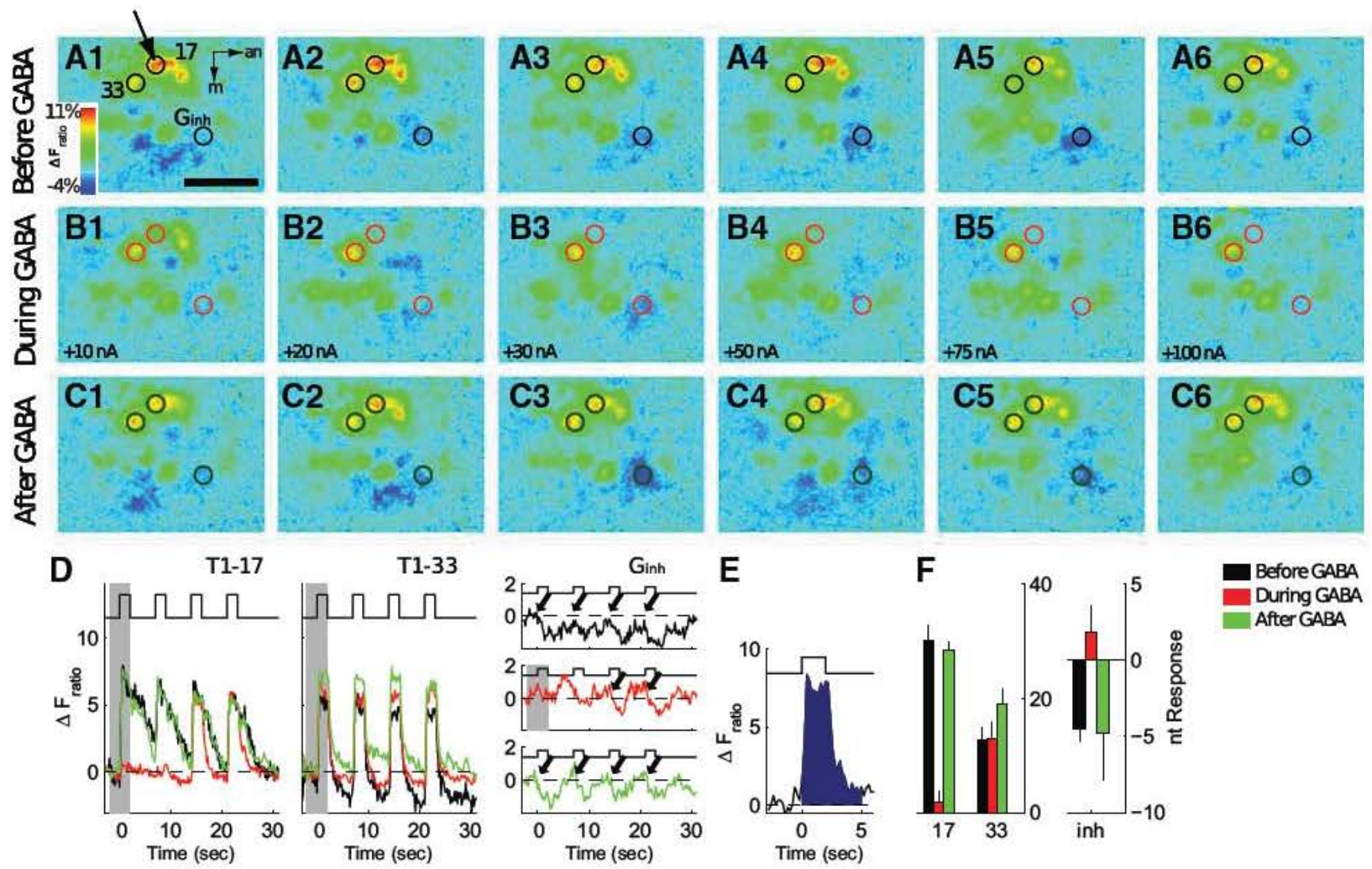

Fig. 3. Interglomerular interactions during GABA injections. $A 1-A 6$ : false-color pictures showing 6 measurements to nonanol stimulation ( 2 s) before GABA injection. Only the response to the first odor pulse is shown. Time traces (with additional odor pulses) of 3 glomeruli (circles) are shown in $D$. Note the inhibition $\left(\mathrm{G}_{\text {inh }}\right)$ triggered by the odor stimulus (e.g., in $A 5$ ). Color bar, directions ( $\mathrm{m}$, medial; an, antennal nerve), and scale bar (100 $\left.\mu \mathrm{m}\right)$ in $A I$ apply to all panels. Arrow in $A 1$, injected glomerulus (T1-17). B1-B6: measurements during odor stimulation ( $2 \mathrm{~s}$, response to first pulse only) and simultaneous GABA injection (4 s). Injection current indicated at bottom left. Otherwise same as A.Cl-C6: measurements after GABA. Otherwise same as A. The odor stimulus triggered the inhibition again in $\mathrm{G}_{\text {inh }}$ (see, e.g., C5). A-C: false-color pictures calculated by subtracting the background response ( 3 frames averaged before stimulus) from the response during odor stimulation ( 3 frames averaged). $D$ : time traces from the 3 glomeruli marked in $A 5, B 5$, and $C 5$ for measurements before (black), during (red, $+75 \mathrm{nA}$, gray bar), and after (green) GABA. The step function (top) shows the 4 odor pulses. For $\mathrm{G}_{\text {inh }}$, traces are averaged ( 4 trials) and plotted separately for better visibility. Note how the inhibition in $\mathrm{G}_{\text {inh }}$ triggered by the odor pulses (shown by arrows) disappeared during GABA injection into T1-17 (gray bar, red trace). Inhibition reappeared after recovery (green trace and 3rd and 4th pulses on red trace). $E$ : example of Int Response measurement. Int Response is the integral (estimated with the trapezoidal method) of time traces from odor onset over $5 \mathrm{~s}$ (blue shaded area). It has units $\% \times \mathrm{s} . F$ : mean responses $(n=4$ trials, error bars are SD). $y$-Axis shows the integrated signal (Int Response) from each of the 3 glomeruli marked in $A, B$, and $C$ before (black), during (red), and after (green) GABA. The response in glomerulus $\mathrm{G}_{\text {inh }}$ is excitatory during GABA injection compared with inhibitory before and after (see text for details). Time traces represent the average of $11 \times 11$ pixels $(27.5 \mu \mathrm{m} \times 27.5 \mu \mathrm{m})$ around the glomerulus center. 
(Fig. 3, $D$ and $F$ ). Recovery after GABA inactivation was fast $(\sim 10 \mathrm{~s}$, Fig. $3 D$, left $)$ and dose dependent.

Odors elicit complex activity patterns across projection neurons in different glomeruli. These activities are driven both by the receptor neuron input and by the AL network (Sachse and Galizia 2002). We therefore asked how a single glomerulus in this pattern influences the responses of other glomeruli. Suppressing one glomerulus did not fundamentally modify the pattern of active glomeruli, which suggests that the excitatory activity in the AL is dominated by receptor neuron input. However, projection neurons also respond with inhibition (calcium decrease) to olfactory stimulation, although this effect is less stereotyped across animals than the excitatory pattern. Note that, because of the low baseline calcium concentration in neurons, inhibitory responses are always small compared with excitatory responses (see below). An example is shown in Fig. 3 , where nonanol consistently and reliably triggered inhibition in a glomerulus (called $G_{\text {inh }}$ in Fig. 3 because not identified). We compared the odor response in glomeruli T1-17, T1-33, and $\mathrm{G}_{\mathrm{inh}}$ before, during, and after injection (2-sample $t$-test, 2-tailed, Bonferroni correction, $P<0.01 / 3$, integrated $5 \mathrm{~s}$ of odor response; see example shown in Fig. $3 E$; mean of measurements with $+30,+50,+75$, and $+100 \mathrm{nA}$, Fig. $3 F$ ). When we injected GABA in T1-17 its odor response was significantly reduced (before vs. during: $t_{6}=17.15, P=2.5 \mathrm{e}-6$ ) and recovered after injection (before vs. after: $t_{6}=1.13, P=0.3$ ). The neighboring glomerulus T1-33 was not significantly influenced by the injection in T1-17 (before vs. during: $t_{6}=0.08$, $P=0.9$; another glomerulus next to T1-17 was also suppressed by GABA with current higher than $+10 \mathrm{nA}$; this might be due to diffusion). Interestingly, the activity in glomerulus $G_{\text {inh }}$ (located on the other side of the AL) was significantly larger (not inhibited anymore) during GABA injection in glomerulus T1-17 (before vs. during: $t_{6}=6.72, P=5.3 \mathrm{e}-4$ ) and recovered after injection (before vs. after: $t_{6}=0.2, P=0.8$ ). These results suggest that there is a direct inhibitory connection from T1-17 to other individual glomeruli in the AL. On the basis of our data we cannot decide whether this unmasked excitation is due to receptor neuron input, to lateral excitation, or to a release from direct inhibition from glomerulus T1-17.

Assuming a direct inhibitory connection, the activation of T1-17 alone (without the remaining odor-evoked pattern) should trigger inhibition in glomerulus $G_{i n h}$. We therefore injected $\mathrm{ACh}(+10$ to $+80 \mathrm{nA}$ ) into T1-17 (via another barrel of the pipette) and indeed found inhibition in glomerulus $\mathrm{G}_{\mathrm{inh}}$ at $+80 \mathrm{nA}$ (data not shown). Thus activity in T1-17 alone was sufficient for the inhibitory response in $\mathrm{G}_{\mathrm{inh}}$. We also observed that ACh induced inhibition in other glomeruli in a patterned manner. This suggests that there are several inhibitory connections from individual glomeruli toward other individual glomeruli within the AL. To map the spatial arrangement of these interglomerular connections, we used local application of $\mathrm{ACh}$ into single glomeruli in subsequent experiments.

Interglomerular inhibition. First, we asked whether inhibition in distant glomeruli depends on the magnitude of the ACh injection. To this end, we varied the injection magnitude (current). We found that the calcium concentration decrease in distant glomeruli was dose dependent. Generally, no inhibition was visible at very low injection magnitudes, although these injections triggered excitation at the injection site (Fig. 4A). In many instances injection of ACh produced inhibitory responses in neighboring glomeruli that were detectable in single trials (Fig. 4B). To assess the reliability of the ACh effect we injected the same current $(+60 \mathrm{nA})$ repeatedly $(n=18)$. Glomeruli showed the same response pattern as in singlecurrent injections (Fig. 4C). In the animal shown in Fig. $4 C$ the excitation in T1-33 triggered inhibition in T1-38 and to a lesser extent in T1-17, while T1-28 was not inhibited. Next, we asked whether the number of inhibited glomeruli increased with larger ACh injections. This analysis was performed on single trials as shown in Fig. $4 B$. For each bee the smallest current that produced significant inhibition (response to ACh: average over $5 \mathrm{~s}$ after injection, significance level: $1.5 \times$ the standard deviation over $4.8 \mathrm{~s}$ before injection) in at least one glomerulus was selected as "low current" and the largest current tested (or $+100 \mathrm{nA}$ if higher currents were used) was set as "high current." The rational for using $1.5 \times$ as threshold is as follows: Calcium decreases due to inhibition are always small because they are limited by calcium pumping capacity of neurons against a background of already low intracellular calcium. Furthermore, background standard deviation is high, because olfactory glomeruli in the bee have a pronounced tendency to be spontaneously active (Galan et al. 2006; see the recording in Supplemental Movie S1 to judge the amount of spontaneous activity in the AL and the inhibitory effect of ACh injection; note in the movie how spontaneous activity decreased during ACh injection and how it recovered within seconds after injection termination). Our settings allowed us to detect reliably small inhibitory effects. For example, the inhibition shown in Fig. $4 D$ is detected by a threshold set at $1.5 \times$ the standard deviation but not by a threshold at $2.5 \times$ the standard deviation. We detected inhibition in 12 bees. On average, low currents produced inhibition in $3 \pm 0.8$ glomeruli (mean $\pm \mathrm{SE}$, median $=2$ ) while high currents inhibited $6.3 \pm 1.0$ glomeruli (mean $\pm \mathrm{SE}$, median $=6.5$ ) per bee (Fig. $4 E$ ). This difference was significant $(n=12$ bees, Wilcoxon signed-rank test, $t=$ $3.5, P=0.0059)$. Using a more conservative significance threshold of $2.5 \times$ the standard deviation for inhibition yielded $0.1 \pm 0.1$ glomeruli with low currents and $3.3 \pm 0.4$ glomeruli with high currents (Wilcoxon signed-rank test, $t=0, P=$ $0.015, n=6$ bees). Therefore, inhibitory connection strength differed among glomeruli: weak injections of ACh led to inhibition in significantly fewer glomeruli than strong injections of $\mathrm{ACh}$ into the same glomerulus. This suggests that inhibitory neural connections between glomerulus pairs are graded and at least partially independent. In our experiments, we never saw evidence for excitatory connections between the injected glomerulus and a distant glomerulus, i.e., excitation (calcium increase) was always limited to the injected glomerulus only or-with a delay (see Fig. $6 C$, inset) - to its direct neighbors, suggesting that these excitations were due to $\mathrm{ACh}$ diffusion rather than to neuronal connectivity.

Inhibition is spatially patchy. What is the spatial organization of the inhibition triggered by activity in a single glomerulus? There are three possible scenarios: 1 ) inhibition could be uniform across the entire AL;2) inhibition could be patchy, but inhibitory strength could be related to distance; or 3) inhibition could be patchy and inhibitory strength not related to distance. First, we generally found that inhibition varied in amplitude and duration in different glomeruli (Fig. 5), indicating that inhibition is not uniform across the AL, and ruling out possibility 1. In Fig. 5 the false-color picture shows the patchy 

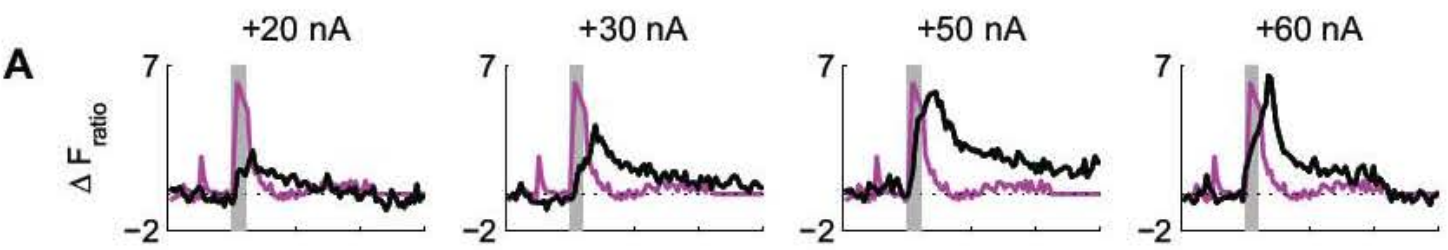

B
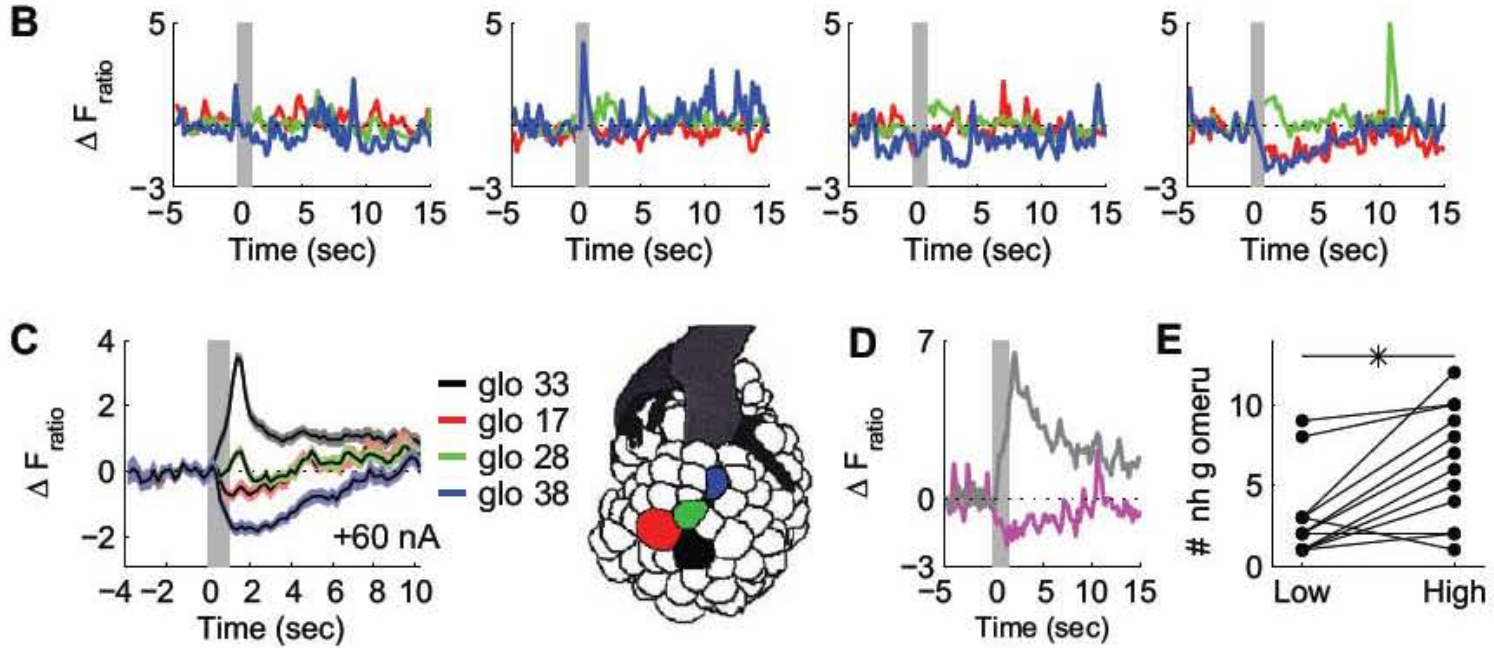

Fig. 4. Response to ACh injections and odors. Example of $\mathrm{ACh}$ injections with different currents. Each injection lasted $1.6 \mathrm{~s}$ (gray bar), and currents indicated apply to $A$ and $B$. A: responses (single trials) to single ACh injections (black) in glomerulus T1-33 (injection site). The purple trace shows the response to 1-nonanol ( $1 \mathrm{~s}$ ) in this glomerulus. $B$ : responses (single trials) of glomeruli T1-17 (red), T1-28 (green), and T1-38 (blue) to ACh injections in T1-33. Note the inhibitory response in T1-17 and T1-38 with $+60 \mathrm{nA}$. The fast and transient calcium increases (e.g., in $B+60 \mathrm{nA}$, green trace, at 11 s) are spontaneous responses (see Galan et al. 2006 and compare Supplemental Movie S1). C: mean response to $18 \mathrm{ACh}$ injections in glomerulus T1-33 (black trace, $1 \mathrm{~s},+60 \mathrm{nA}$, gray bar). The mean responses in glomeruli T1-17 (red), T1-28 (green), and T1-38 (blue) as a result of the injections in T1-33 are also shown. Note how excitation and inhibition started simultaneously. Shading shows the $\mathrm{SE}(n=18$ trials). The AL schematic shows the glomeruli position in colors matching the traces in $A, B$, and $C . D$ : example from another AL showing the calcium response in the injected glomerulus (gray trace, $1.6 \mathrm{~s},+80 \mathrm{nA}$, gray bar) and in an unidentified inhibited glomerulus (pink trace). In this example a threshold of $2.5 \times$ the standard deviation would not detect inhibition due to high spontaneous activity before the injection. $E$ : each pair represents a bee $(n=12)$ with the number of significantly inhibited glomeruli (threshold: $1.5 \times$ standard deviation, see text for details) with the lowest current ("Low" on $x$-axis) and the highest current ("High" on $x$-axis) tested. Low current varied from +20 to $+50 \mathrm{nA}$ and high current from +40 to $+100 \mathrm{nA}$ depending on the bee. The difference between both groups was significant (Wilcoxon signed-rank test, $* P=0.0059$ ).

pattern triggered by the injection of $\mathrm{ACh}$. To distinguish between the other two possibilities, we quantified the spatial pattern of interglomerular inhibition and asked how the strength of inhibition depended on the distance to the injection pipette. We plotted signal amplitude produced by the injection against the distance to the injection site for six bees (Fig. 6A). Significant signal changes were mostly inhibitory (48/59 glomeruli, Fig. 6, $B$ and $C$ ). Excitatory ACh responses were only found close to the injection site and were likely caused by diffusion (Fig. $6 \mathrm{C}$ ). In those cases they were delayed (Fig. $6 \mathrm{C}$, inset). The mean distance to pipette of excitatory $\mathrm{ACh}$ responses $(46.2 \pm 6.9 \mu \mathrm{m})$ was significantly smaller than the mean distance to pipette of the inhibitory $\mathrm{ACh}$ responses $\left(109.8 \pm 4.9 \mu \mathrm{m}\right.$, mean $\pm \mathrm{SE}$; 2 -sample $t$-test, 2-tailed, $t_{57}=$ $5.94, P=1.8 \mathrm{e}-7)$. The amplitudes of inhibition were never correlated with distance $(-0.48 \leq r \leq 0.58,0.23 \leq P \leq 0.71$; Fig. $6 A$ ). Analysis of normalized and pooled data showed no relationship between distance to injected glomerulus and in-

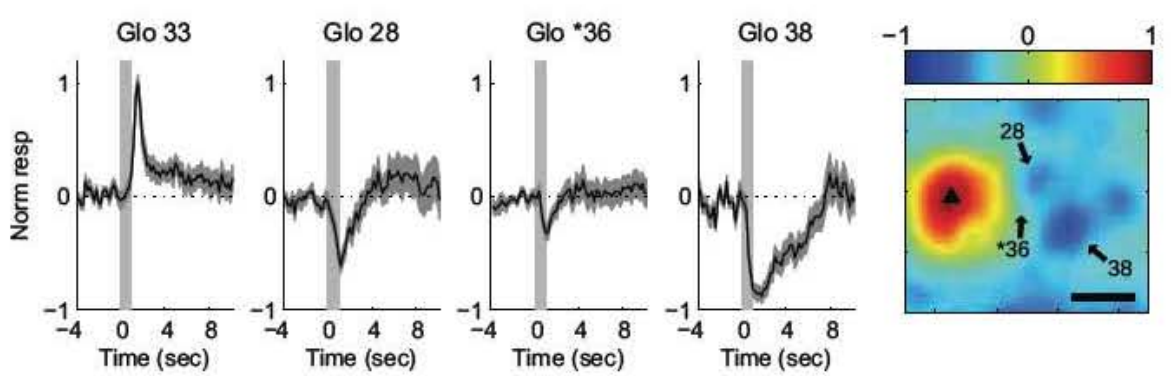

Fig. 5. ACh injections trigger inhibition of different amplitudes and durations in the AL. Traces show the normalized responses (normalization relative to peak of response at the injection site in T1-33) of 4 example glomeruli. Vertical gray bar shows the ACh injection. Gray shading shows SE (18 trials). Note how inhibition varies in both amplitude and duration depending on the glomerulus. T1-36 was tentatively identified (denoted by asterisk). The false-color picture (triangle shows glomerulus T1-33, the injected glomerulus) represents the mean (18 trials) normalized response during a ACh injection. The false-color picture was calculated by averaging 18 trials [each trial is the average of 2 frames after injection termination with background subtracted (average of 35 frames before injection), see MATERIALS AND METHODS]. Note that, in false-color pictures, glomerulus size depends on activity. Time traces represent the average of $11 \times 11$ pixels $(27.5 \mu \mathrm{m} \times 27.5 \mu \mathrm{m})$ around the glomerulus center. Scale bar, $50 \mu \mathrm{m}$. Antennal nerve is left and lateral is top. 
Fig. 6. Inhibition and excitation vs. distance to injection site. $A$ : responses plotted as difference in Int Resp (Int Resp during injection - Int Resp before injection) against the distance to the injection pipette. Int Resp was calculated as in Fig. $3 E$ (but for injection instead of odor). Each of the 6 plots shows data from 1 bee. Glomeruli with a significant change (black circles) and a nonsignificant change (gray squares) are shown. Significance was assessed with 2 -sample $t$-tests (signal before injection was compared with signal during injection) and Bonferroni-Holm correction for multiple comparisons. Injections lasted $1 \mathrm{~s}$ (12-24 trials). $B$ : pooled data for all bees $(n=6)$ from $A$, each bee normalized to its largest inhibition (set to -1 ). Symbol shapes and colors are as in $A$. In $A$ and $B$ glomeruli with large excitatory response (close to injection site) are out of $y$-axis range (all are shown in $C$ ). $C$ : same data as $B$, but only significant glomeruli (black circles in $B$ ) are inhibitory responses are shown with triangles and dots, respectively (see text for details). Inset: time course example of a significantly excited glomerulus (thick line, at $80 \mu \mathrm{m}$ from injection pipette) compared with a significantly inhibited glomerulus (thin line) located further away $(127 \mu \mathrm{m})$ from the injection pipette. Note the delayed excitatory response after injection (gray bar). Each trace is the mean of 18 trials; gray shading shows SE. $x$-Axis unit: time (s); $y$-axis unit: $\mathrm{dF} / \mathrm{F}(\%)$. plotted and $y$-axis is set to show all data points. Excitatory and
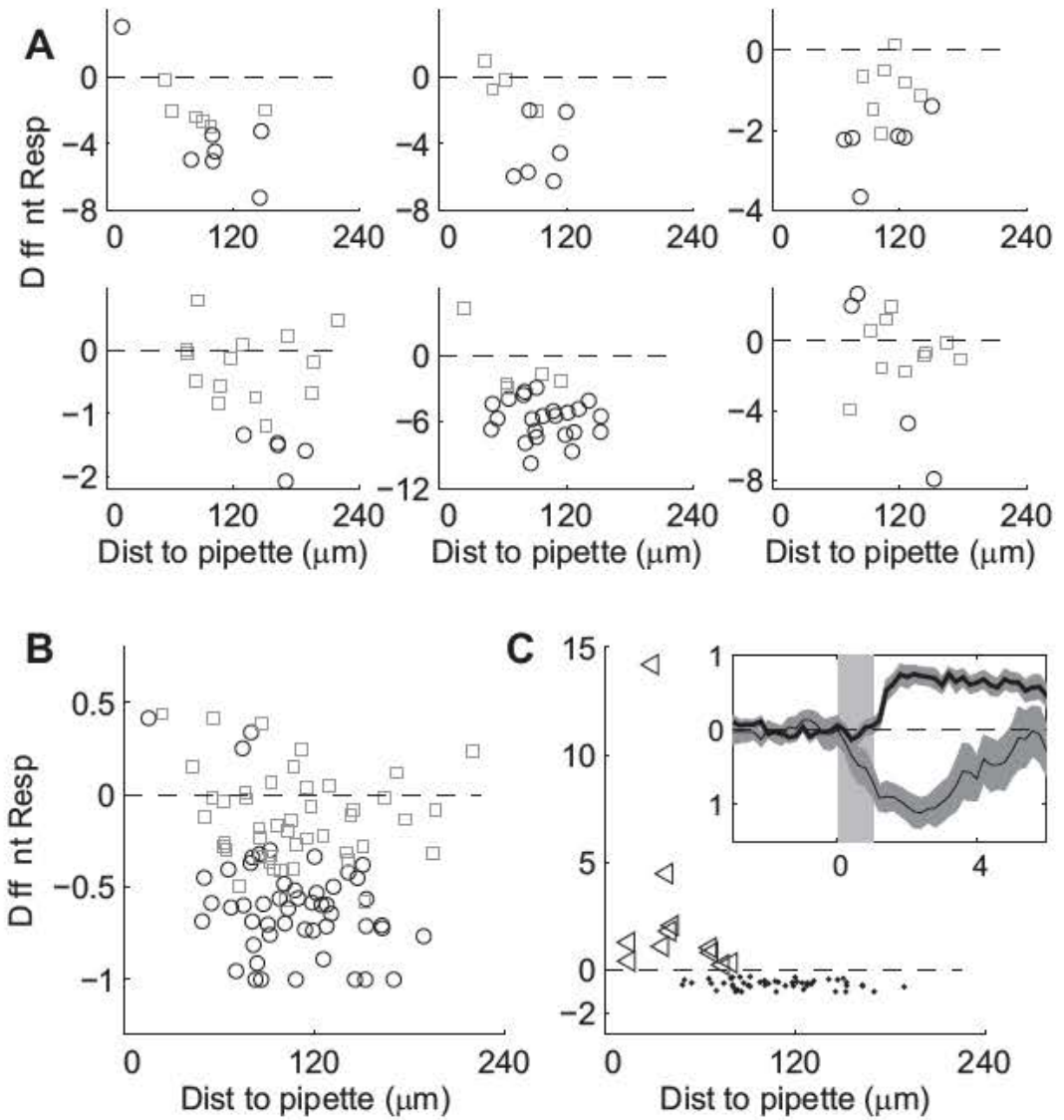

hibitory strength $(r=-0.16, P=0.28$; Fig. $6 B)$. These data are based on repeated injections of the same current. A similar analysis was performed on another set of experiments $(n=14$ bees, data not shown) with single injections of different currents, confirming the finding that the inhibition triggered in remote glomeruli was not correlated with distance $(r=0.14$, $P=0.30)$. Because we systematically targeted the same glomeruli (T1-17 or T1-33) in each animal, we could also ask whether the inhibitory connectivity pattern was equal across individuals; we found no consistent pattern (see DISCUSSION).

Inhibition strength differs among glomeruli. Together, the data show that each glomerulus inhibits a patchy pattern of glomeruli across the AL (Figs. 5 and 6) and that inhibitory strength varies for different glomeruli (Figs. 4-6). To test whether glomeruli receive inhibitory input from a patchy group of glomeruli, we injected $\mathrm{ACh}$ at two locations in the same $\mathrm{AL}$. We observed that some glomeruli received inhibition from only one location (Fig. 7, $A$ and $B$ ) while others received inhibition from both locations (Fig. 7, $C$ and $D$ ). This indicates that the presence of inhibition is not a property of the receiving glomerulus but rather of the connection between a specific glomerulus pair.

Different levels of inhibition could be due either to different connection strengths or to intrinsic properties of the receiving (inhibited) glomerulus. For example, glomeruli differ in their level of background activity, affecting their resting calcium level. This property may be intrinsic or inherited from different levels of background activity in their respective receptor neuron classes. A glomerulus in which projection neurons have a very high resting calcium level will show a strong calcium decrease when inhibited, while a glomerulus in which projection neurons have a low resting calcium level will show a small calcium decrease. Thus apparent inhibition depends not only on connectivity but also on the projection neurons being measured (note that this observation is independent of the measurement technique and would equally apply to spike counts or excitatory postsynaptic potential size in electrophysiological measurements). Therefore, in order to analyze whether inhibitory strength depends on glomerular distance, intrinsic properties of the receiving glomerulus need to be excluded. To this end, we calculated the ratio of the responses for glomeruli showing a negative deflection during injection at two locations (Fig. 7E). Calculating this ratio removes the effect of intrinsic properties of the receiving glomerulus, e.g., of different resting calcium levels that might be due to intrinsic properties or to tonic levels of spontaneous activity. Therefore, if we do not find a systematic distance relationship in the ratio of the two signals, inhibitory connections do not systematically decrease with glomerular distance, adding to their patchy connectivity logic. For illustration, we show a hypothetical example in Fig. 8: a distance-dependent inhibition that is not visible in the measured signal because of added variability becomes apparent when calculating the ratio and plotting it against the relative distance (Fig. 7E). Our measurements in honeybees show that the ratio of the inhibition did not depend on the relative distance between the injection location and the inhibited glomerulus (Fig. 7E). Thus both patchiness and different inhibitory strengths are properties of the inhibitory network, and not dictated by the geometry of the AL. Most importantly, there is no correlation between the amplitude of the inhibition received by a glomerulus and the distance to the source of this inhibition (injection site). 

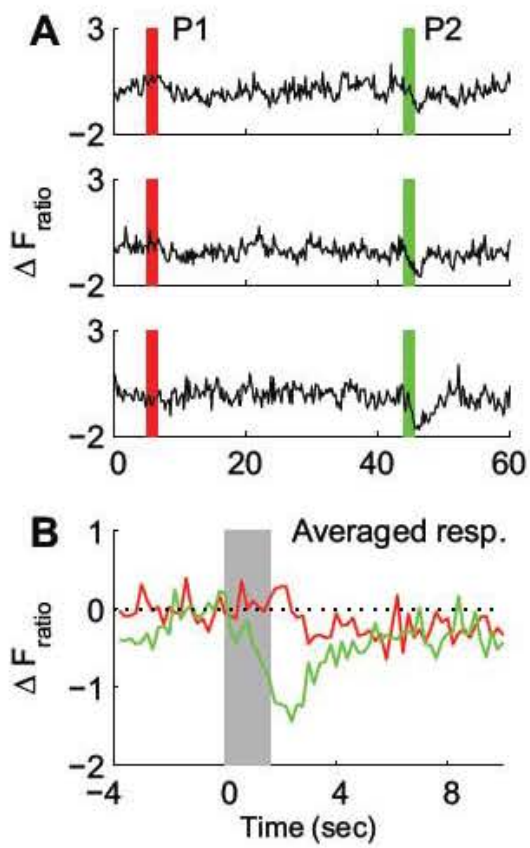
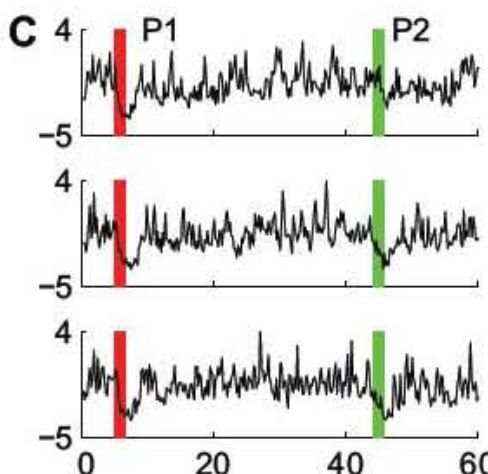

$D_{2}$

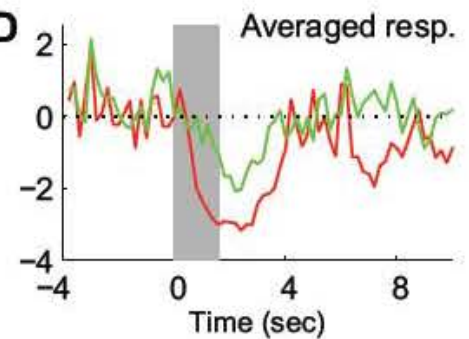

Fig. 7. ACh injection at 2 locations in the same AL. A: time traces of responses in a glomerulus remote from both injection sites. Single trials are shown. The injection at the first location (P1, red, at time $5 \mathrm{~s}$ ) did not produce inhibition, while the other location (P2, green, at time $44 \mathrm{~s}$ ) triggered inhibition in that glomerulus. $B$ : averaged responses $(n=3)$ from injections at P1 (red) and at P2 (green) from data shown in $A$. C: response of another glomerulus from the same bee; otherwise as in $A$. Note that this glomerulus received inhibition from P1 and from P2. $D$ : averaged responses $(n=3)$ from injections at P1 (red) and at P2 (green) from data shown in $C$. Both locations induced inhibition. $E$ : response ratio of each glomerulus plotted against the distance difference; total of 18 glomeruli ( 2 bees). Yellow squares and line show how the data points would distribute if the inhibition depended on distance as in the hypothetical example of Fig. 8.

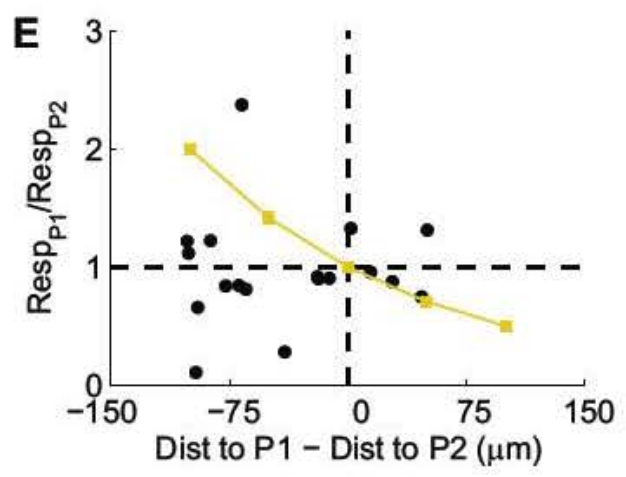

Odor responses are affected by the inhibitory network. These measurements show that interglomerular inhibition can decrease activity in projection neurons. Does this inhibition also modify the spatial activity pattern elicited by stimulation with odors? We combined ACh injections $(1.6 \mathrm{~s},+30$ to +80 $\mathrm{nA}$ ) with odor stimulations ( $1 \mathrm{~s})$. We found that injection of $\mathrm{ACh}$ into a glomerulus during odor presentation suppressed the odor responses of projection neurons in other glomeruli (Fig. 9,<smiles>Cc1cccc(-c2ccccc2)c1</smiles>
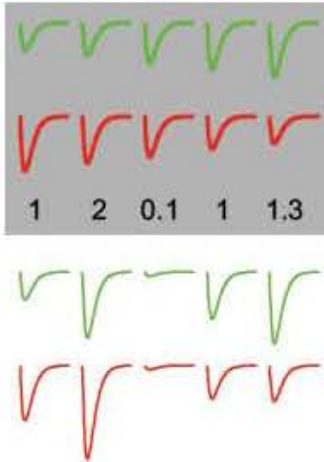

Real inhibition when injecting green glomerulus $\longrightarrow$ Distance dependent

Real inhibition when injecting red glomerulus $\rightarrow$ Distance dependent

Unspecific factor modifying the measured signal e.g. due to different resting calcium level

Measured inhibition when injecting green glomerulus $\longrightarrow$ Appears as distance independent

Measured inhibition when injecting red glomerulus $\longrightarrow$ Appears as distance independent

Reconstrucion of distance dependent inhibition after calculating the ratio between both measured signals.
Fig. 8. Hypothetical case of distance-dependent inhibition. Cartoon shows 2 injected glomeruli (red and green circles with pipette) and 5 inhibited glomeruli (black circles). The real inhibition triggered by $\mathrm{ACh}$ injections decreases with distance (thick red and green lines). However, local factors (e.g., different resting calcium level in each glomerulus) can modify (here by multiplication) the measured signal so that the distance dependence of inhibition is not visible (thin red and green lines). Note that none of the parameters (real inhibition and unspecific factors) in the gray box can be estimated experimentally. Nevertheless, we can remove local effects by calculating the ratio of the measured signals. This reveals the distance dependence of inhibition for this hypothetical example (yellow histograms). This is also plotted in Fig. 7 with yellow squares for comparison with real data. 
A

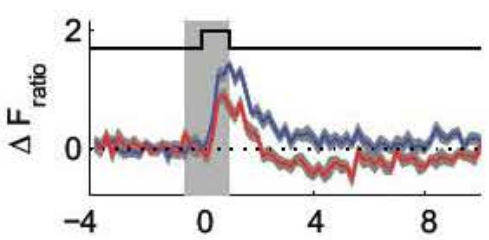

Fig. 9. ACh injections combined with odor stimulation. $A$, bottom: response of a glomerulus to $\mathrm{ACh}$ injection into another glomerulus $(+30 \mathrm{nA}$, mean of 18 trials, no odorant). Note the inhibition in this glomerulus from lateral connectivity. Top: mean responses in the same glomerulus $(n=12)$ to an odorant alone (blue) and to an odorant presented together with $\mathrm{ACh}$ application into the injected glomerulus (red). The inhibition produced by $\mathrm{ACh}$ reduced the odor response. The step function represents the odor ( $1 \mathrm{~s}$ ), and the gray bar represents the $\mathrm{ACh}$ injection into the injected glomerulus $(1.6 \mathrm{~s},+30 \mathrm{nA})$. Gray shading is SE. $B$ and $C: 2$ other example glomeruli with odor response suppression when injecting $\mathrm{ACh}$ into another glomerulus; otherwise as in $A$. D: scatterplot of inhibition (integrated response) vs. response suppression. Both variables are correlated ( $r=-0.71, P=1.1 \mathrm{e}-5$; note the reversed $y$-axis, hence the negative correlation coefficient despite a positive slope), suggesting that the reduced odor response is indeed caused by inhibitory interglomerular connections activated by $\mathrm{ACh}$ injection. Each cross is a glomerulus with inhibition triggered by an $\mathrm{ACh}$ injection ( 30 glomeruli from 4 bees). Note that some glomeruli had a strong odor response reduction although they had only a small inhibitory response to injection alone (see text for discussion).

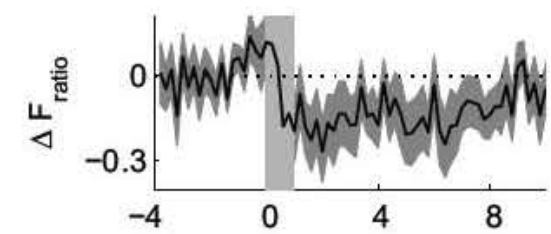

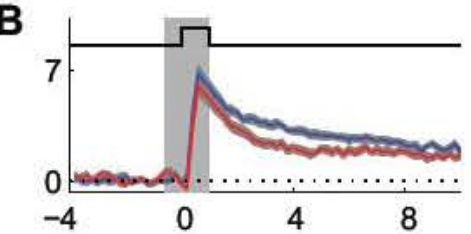
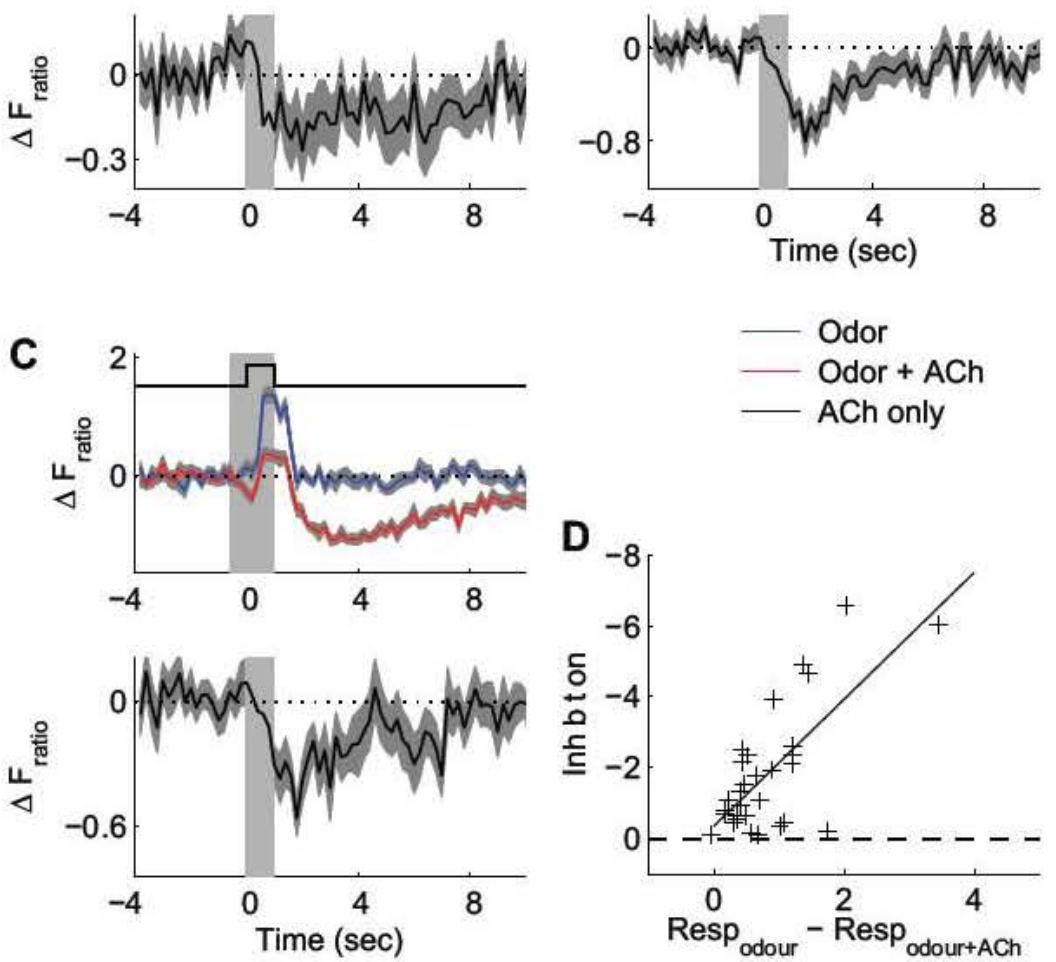

$A-C$ ). Thus inhibition elicited by localized $\mathrm{ACh}$ injections influenced the activity triggered by the odorant. This effect was quantitatively related to the interglomerular inhibitory strength (Fig. 9D). To show this, we compared the inhibition produced by $\mathrm{ACh}$ injection alone ( $1 \mathrm{~s})$ to the odor suppression observed when combining injection and odor stimulation (1.6-s ACh injection and 1-s odor stimulation). To make sure that the inhibition triggered by the injection was present at odor onset, we started the ACh injection $0.6 \mathrm{~s}$ before the odor. Injection and odor stimulation terminated simultaneously (Fig. 9, $A-C$ ). Because the $\mathrm{ACh}$ injection was longer in one case and because the odor stimulation can recruit inhibition (see Fig. 3), it is likely that the total inhibition present in both situations (injection alone or injection + odor) was different. We found that the inhibition (elicited by $\mathrm{ACh}$ injection alone) and the response suppression (measured by combining injection and odor stimulus) were correlated (Fig. $9 D ; r=-0.71, P=1.1 \mathrm{e}-5$ ). We thus conclude that the interglomerular inhibitory network analyzed here is directly involved in processing odor-evoked responses.

Local neurons have patchy morphology. A patchy connectivity could be achieved by morphologically homogeneous neurons with different synaptic densities in different glomeruli or by morphologically patchy neurons. To investigate the morphology of local neurons connected with a single glomerulus, we visualized them by iontophoretic application of the tracer biocytin into a single glomerulus. We found that several local neurons were labeled, some of which were heterogeneous local neurons and some homogeneous local neurons (an example is given in Fig. 10).

\section{DISCUSSION}

In this study we have manipulated the AL circuit with GABA and ACh, two abundant neurotransmitters in the AL (Kreissl and Bicker 1989; Schäfer and Bicker 1989). Our method using local injections takes advantage of directly activating or inactivating an identified glomerulus in vivo. Our goal was to examine the role of a glomerulus within this olfactory network. First, our calcium imaging results confirmed the excitatory effect of ACh (Waldrop and Hildebrand 1989) and the inhibitory effect of GABA (Michelsen and Braun 1987 ) in the insect AL. Second, we have shown that activating a glomerulus triggered inhibition in spatially scattered glomeruli. Furthermore, the strength of inhibition between glomeruli did not depend on the distance between them. This has important consequences for the computation in the olfactory system. Finally, we found that the interglomerular inhibitory connections revealed by $\mathrm{ACh}$ injections can modulate an odor response.

Technical considerations. To minimize undesired pharmacological effects (e.g., seizures), we chose to only use neurotransmitters already present in the brain to manipulate the 

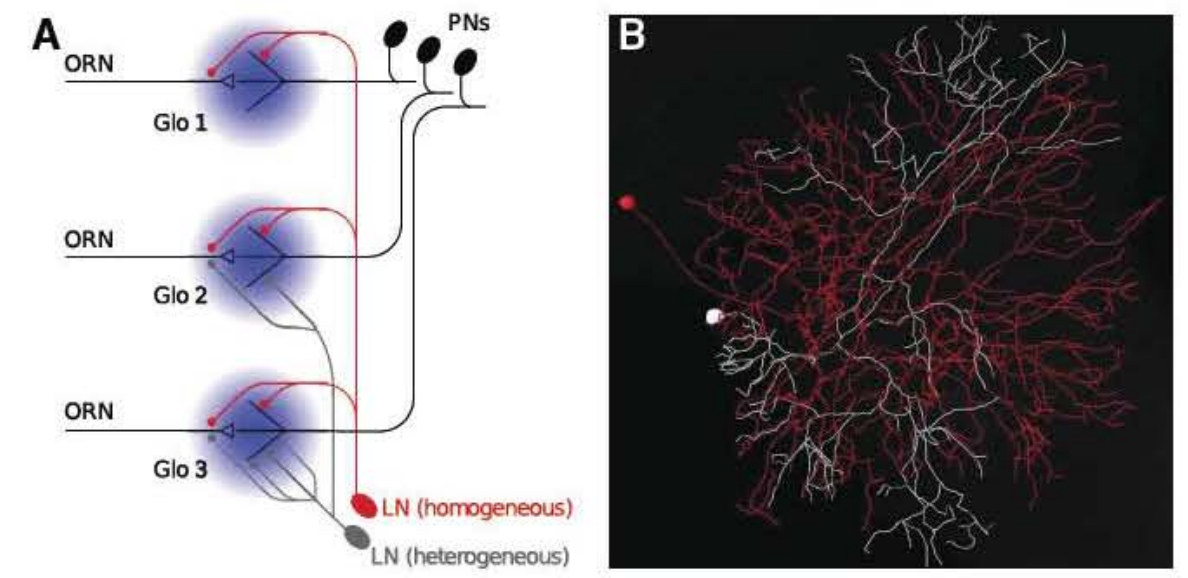

Fig. 10. A: schematic circuit of the AL showing 3 glomeruli in blue (Glo 1, Glo 2, and Glo 3). Each neuron in the schematic represents a population of neurons in vivo. Each glomerulus receives input from olfactory receptors (ORNs) and produces output via projection neurons (PNs). Glomeruli also receive inhibition from homogeneous (red) and heterogeneous (gray) local interneurons (LN). The homogeneous local neuron innervates each glomerulus equally, while the heterogeneous local neuron forms many synapses only in Glo 3 and no synapse in Glo 1. Only the most important connections are included in the cartoon, and the inhibitory synapses (red and gray dots) can, in principle, be GABAergic or use a different transmitter (e.g., histaminergic). In our experiment, activating the gray heterogeneous local neuron with ACh would trigger different amounts of inhibition in Glo 1,2 , and 3 . This would be reflected in the PNs with strong inhibition in Glo 3, moderate inhibition in Glo 2, and no inhibition in Glo 1. B: reconstruction of a homogeneous (red) and a heterogeneous (gray) local neuron in the AL of the honeybee. The somata of these neurons are on left. Note that the heterogeneous local neuron (gray) is well suited to produce patchy inhibition across the $\mathrm{AL}$ since it forms connections in a restricted number of glomeruli.

network. Several pieces of evidence in our data suggest that the AL was not disrupted beyond the activity at the injection site. First, with $\mathrm{ACh}$ injections of the amplitude used here the excitation remained localized and we never observed excitation in remote glomeruli. Second, while ACh injections produce relatively long-lasting responses (several seconds), odors do as well (compare Fig. $4 A$ and Fig. 9B). This, in fact, shows that $\mathrm{ACh}$ injections are likely to mimic an odor that would activate a single glomerulus. Finally, the response pattern to odors during a GABA or $\mathrm{ACh}$ injection is completely normal in glomeruli outside of the injection site (except for the additional lateral inhibition; see Figs. 3 and 9). Therefore, we are confident that our injections did not produce dysfunctional activity in the AL.

Spatial organization of lateral inhibitory connections. Population analyses of electrophysiologically recorded cells have suggested that the macroglomerular complex (special glomeruli for pheromone processing in moths) inhibits other glomeruli in a distance-independent manner (Reisenman et al. 2008). Our imaging technique in honeybees, which allows us to record simultaneously the activity in multiple glomeruli in an organism lacking a macroglomerular complex, also revealed distance-independent inhibition. This principle might therefore represent a fundamental connectivity rule of the AL. In the olfactory bulb of vertebrates, however, distance-dependent and distant-independent interactions have been reported (see, e.g., Aungst at al. 2003, Fantana et al. 2008; Kim et al. 2011, 2012; Luo and Katz 2001; Vucinic et al. 2006; Yokoi et al. 1995). Interestingly, computer simulations using distance-independent inhibition can reproduce physiological data (Cleland and Sethupathy 2006; Linster et al. 2005). Here, we directly measured the strength of inhibition simultaneously at several locations, using calcium imaging. Our results show that the lateral inhibitory network at the first stage of olfactory processing in the honeybee is patchy, and both the presence and the strength of inhibition between two glomeruli cannot be predicted by their relative distance. Furthermore, comparing across individuals we did not find a predictable pattern of inhibited glomeruli. While part of this could be due to difficulties in glomerular identification, it appears that, unlike the very stereotypical excitatory pattern elicited by odors, the inhibitory connectivity matrix is more variable across animals, as recently reported in Drosophila (Chou et al. 2010). This may reflect modifications by developmental plasticity and/or learning in the adult. The effect of local networks is best visible in processing of odor-mixture stimuli (e.g., Deisig et al. 2006; Silbering and Galizia 2007) and olfactory learning effects (Fernandez et al. 2009; Rath et al. 2011). A scattered connectivity may be the substrate for and/or the consequence of this plasticity. The results mentioned above and our results confirm the hypothesis that the olfactory network map (and possibly other maps in the brain) results from a combination of hard-wired, templated (e.g., olfactory receptor neurons to projection neuron in a glomerulus connection), and plastic, more diverse connections (e.g., the lateral inhibitory connections shown in this study). Figure $10 \mathrm{~A}$ shows a cartoon of the AL network including only the most relevant connections. Inhibition between glomeruli is provided by two anatomical types of local neurons: heterogeneous and homogeneous local neurons (Flanagan and Mercer 1989; Fonta et al. 1993; Sun et al. 1993). Activating a heterogeneous local neuron in Fig. $10 \mathrm{~A}$ with $\mathrm{ACh}$ would inhibit the three glomeruli differently since they receive different amounts of inhibition from that local neuron. Figure $10 B$ shows two reconstructed local neurons from one AL. Local neurons such as the gray neuron (heterogeneous) in Fig. $10 B$ are likely to represent the anatomical substrate of the physiological effect reported in the present study. In other words, a patchy anatomical structure of heterogeneous local neurons might contribute to the patchy lateral inhibition observed in this study. Alternatively, homogeneous local neurons could also contribute if they were functionally heterogeneous. Note that both $\mathrm{GABA}_{\mathrm{A}}$ and $\mathrm{GABA}_{\mathrm{B}}$ inhibition have been reported in the AL of insects (e.g., Christensen et al. 1998a; Olsen and 
Wilson 2008) and that both might be involved here. Another potent inhibitory neurotransmitter in the honeybee is histamine (Sachse et al. 2006). Addressing questions about which inhibitory neurotransmitter(s) was involved in the effect we observe is beyond the scope of this work. Also, the effects reported here might be the result of monosynaptic or polysynaptic pathways.

Implications for odor processing. From a developmental and efficiency point of view, a network should be optimized for total connectivity length, as shown, for example, with the development of ocular dominance stripes in the mammalian visual cortex (e.g., Mitchison 1992), unless there are constraints with respect to temporal delay lines. Given the small size and spherical arrangement of the bee AL, total connectivity length appears to be the main factor. Therefore, we would expect that glomeruli that are interconnected should tend to be neighbors. The fact that our data prove this assumption wrong suggests that olfactory processing requires strategies that are compatible with the constraints of the olfactory space (see Cleland 2010). This might also influence another peculiarity of the bee AL: most local neurons enter the core of the AL, but all glomeruli are arranged as a spherical sheet in the periphery. In this arrangement, all glomeruli are topologically equidistant from the AL center, an ideal arrangement if there is no two-dimensional logic for their position.

Interestingly, we only detected inhibitory interactions (except close to or at injection site), although excitatory connections exist in Drosophila (Olsen et al. 2007; Root et al. 2007; Shang et al. 2007). This suggests that the lateral network might be dominated by inhibition in honeybees. Up to now, no excitatory local neurons have been shown in insects other than Drosophila.

Despite excitatory and inhibitory interglomerular interactions, it has been proposed that receptor neuron input constitutes the dominating excitatory signal onto projection neurons (Sachse and Galizia 2003; Root et al. 2007). Our GABA injection experiments showed that the excitatory response pattern to odors was relatively stable (except for the injected glomerulus) when one glomerulus was pharmacologically silenced. Furthermore, glomeruli inhibited via lateral inhibition with ACh injections still responded, albeit less, to the odor. These two observations support a model in which a strong input imposes the spatial response pattern while the global activity of the network modulates, mainly via lateral inhibition, the response of the glomeruli. Nevertheless, one should consider that data (present study and others) were obtained by using squared pulse odor stimulation of relatively high concentrations. Under natural stimulation conditions (fluctuating odor mixtures at low concentrations) the influence of the patchy inhibitory network on spatiotemporal activity patterns in the AL is likely to become more important. For example, in response to dynamic stimuli projection neurons produce reliable responses across trials, and these responses are odor specific (Geffen et al. 2009). Furthermore, the temporal patterns of these responses depend on GABAergic inhibition (Christensen et al. 1998b). The patchy inhibitory network described in the present work is ideally placed to play a role in such computation.

Conclusion. In summary, we show that the bee AL inhibitory network is structured in a complex spatial arrangement, following a logic that is not dictated by geometrical position in the AL. This network is capable of significantly shaping odor responses. Whether, and how, this patchy arrangement is used for odor-mixture coding, and how it is influenced by olfactory learning, remain to be elucidated.

\section{ACKNOWLEDGMENTS}

We thank the members of the Galizia lab and particularly Corinna Geiss for anatomical reconstruction and Dr. Henning Proske for critical comments on the manuscript.

\section{DISCLOSURES}

No conflicts of interest, financial or otherwise, are declared by the author(s).

\section{AUTHOR CONTRIBUTIONS}

Author contributions: C.C.G. and C.G.G. conception and design of research; C.C.G. and S.K. performed experiments; C.C.G. analyzed data; C.C.G. and C.G.G. interpreted results of experiments; C.C.G. prepared figures; C.C.G., S.K., and C.G.G. drafted manuscript; C.C.G., S.K., and C.G.G. edited and revised manuscript; C.C.G., S.K., and C.G.G. approved final version of manuscript.

\section{REFERENCES}

Aungst JL, Heyward PM, Puche AC, Karnup SV, Hayar A, Szabo G, Shipley MT. Centre-surround inhibition among olfactory bulb glomeruli. Nature 426: 623-629, 2003.

Chou YH, Spletter ML, Yaksi E, Leong JC, Wilson RI, Luo L. Diversity and wiring variability of olfactory local interneurons in the Drosophila antennal lobe. Nat Neurosci 13: 439-449, 2010.

Christensen TA, Waldrop BR, Hildebrand JG. GABAergic mechanisms that shape the temporal response to odors in moth olfactory projection neurons. Ann NY Acad Sci 855: 475-481, 1998a.

Christensen TA, Waldrop BR, Hildebrand JG. Multitasking in the olfactory system: context-dependent responses to odors reveal dual GABA-regulated coding mechanisms in single olfactory projection neurons. J Neurosci 18: 5999-6008, 1998b.

Cleland TA. Early transformations in odor representation. Trends Neurosci 33: $130-139,2010$.

Cleland TA, Sethupathy P. Non-topographical contrast enhancement in the olfactory bulb. BMC Neurosci 7: 1-18, 2006.

Deisig N, Giurfa M, Lachnit H, Sandoz JC. Neural representation of olfactory mixtures in the honeybee antennal lobe. Eur J Neurosci 24: 1161-1174, 2006

Distler PG, Boeckh J. An improved model of the synaptic organization of insect olfactory glomeruli. Ann NY Acad Sci 855: 508-510, 1998.

Fantana AL, Soucy ER, Meister M. Rat olfactory bulb mitral cells receive sparse glomerular inputs. Neuron 59: 802-814, 2008

Fernandez PC, Locatelli FF, Person-Rennell N, Deleo G, Smith BH. Associative conditioning tunes transient dynamics of early olfactory processing. J Neurosci 29: 10191-10202, 2009.

Flanagan D, Mercer A. Morphology and response characteristics of neurones in the deutocerebrum of the brain in the honeybee Apis mellifera. J Comp Physiol A 164: 484-494, 1989.

Fonta C, Sun X, Masson C. Morphology and spatial distribution of bee antennal lobe interneurones responsive to odors. Chem Senses 18: 101-119, 1993.

Galan RF, Weidert M, Menzel R, Herz AV, Galizia CG. Sensory memory for odors is encoded in spontaneous correlated activity between olfactory glomeruli. Neural Comput 18: 10-25, 2006.

Galizia CG, Kimmerle B. Physiological and morphological characterization of honeybee olfactory neurons combining electrophysiology, calcium imaging and confocal microscopy. J Comp Physiol A Neuroethol Sens Neural Behav Physiol 190: 21-38, 2004.

Geffen MN, Broome BM, Laurent G, Meister M. Neural encoding of rapidly fluctuating odors. Neuron 26: 570-786, 2009.

Kim DH, Chang AY, McTavish TS, Patel HK, Willhite DC. Centersurround vs. distance-independent lateral connectivity in the olfactory bulb. Front Neural Circuits 6: 34, 2012. 
Kim DH, Phillips ME, Chang AY, Patel HK, Nguyen KT, Willhite DC. Lateral connectivity in the olfactory bulb is sparse and segregated. Front Neural Circuits 5: 5, 2011.

Kreissl S, Bicker G. Histochemistry of acetylcholinesterase and immunocytochemistry of an acetylcholine receptor-like antigen in the brain of the honeybee. J Comp Neurol 286: 71-84, 1989.

Kreissl S, Strasser C, Galizia CG. Allatostatin immunoreactivity in the honeybee brain. J Comp Neurol 518: 1391-1417, 2010.

Linster C, Sachse S, Galizia CG. Computational modeling suggests that response properties rather than spatial position determine connectivity between olfactory glomeruli. J Neurophysiol 93: 3410-3417, 2005.

Luo M, Katz LC. Response correlation maps of neurons in the mammalian olfactory bulb. Neuron 32: 1165-1179, 2001.

Michelsen DB, Braun GH. Circling behavior in honey bees. Brain Res 421: 14-20, 1987.

Mitchison G. Axonal trees and cortical structures. Trends Neurosci 15: 122-126, 1992.

Müller D, Abel R, Brandt R, Zöckler M, Menzel R. Differential parallel processing of olfactory information in the honeybee, Apis mellifera l. J Comp Physiol A Neuroethol Sens Neural Behav Physiol 188: 359-370, 2002.

Olsen SR, Bhandawat V, Wilson RI. Excitatory interactions between olfactory processing channels in the Drosophila antennal lobe. Neuron 54: 89-103, 2007.

Olsen SR, Wilson RI. Lateral presynaptic inhibition mediates gain control in an olfactory circuit. Nature 452: 956-960, 2008.

Rath L, Galizia CG, Szyszka P. Multiple memory traces after associative learning in the honey bee antennal lobe. Eur J Neurosci 34: 352-360, 2011.

Reisenman CE, Heinbockel T, Hildebrand JG. Inhibitory interactions among olfactory glomeruli do not necessarily reflect spatial proximity. $J$ Neurophysiol 100: 554-564, 2008.

Root CM, Semmelhack JL, Wong AM, Flores J, Wang JW. Propagation of olfactory information in Drosophila. Proc Natl Acad Sci USA 104: $11826-$ 11831, 2007.

Sachse S, Galizia CG. Role of inhibition for temporal and spatial odor representation in olfactory output neurons: a calcium imaging study. Neurophysiol 87: 1106-1117, 2002.
Sachse S, Galizia CG. The coding of odour-intensity in the honeybee antennal lobe: local computation optimizes odour representation. Eur J Neurosci 18: 2119-2132, 2003

Sachse S, Peele P, Silbering AF, Gühmann M, Galizia CG. Role of histamine as a putative inhibitory transmitter in the honeybee antennal lobe. Front Zool 3: 22, 2006.

Sachse S, Rappert A, Galizia CG. The spatial representation of chemical structures in the antennal lobe of honeybees: steps towards the olfactory code. Eur J Neurosci 11: 3970-3982, 1999.

Schäfer S, Bicker G. Distribution of GABA-like immunoreactivity in the brain of the honeybee. J Comp Neurol 246: 287-300, 1986.

Shang Y, Claridge-Chang A, Sjulson L, Pypaert M, Miesenböck G. Excitatory local circuits and their implications for olfactory processing in the fly antennal lobe. Cell 128: 601-612, 2007.

Silbering AF, Galizia CG. Processing of odor mixtures in the Drosophila antennal lobe reveals both global inhibition and glomerulus-specific interactions. J Neurosci 27: 11966-11977, 2007.

Sun X, Fonta C, Masson C. Odour quality processing by bee antennal lobe neurons. Chem Senses 18: 355-377, 1993.

Szyszka P, Ditzen M, Galkin A, Galizia CG, Menzel R. Sparsening and temporal sharpening of olfactory representations in the honeybee mushroom bodies. J Neurophysiol 94: 3303-3313, 2005.

Vucinic D, Cohen LB, Kosmidis EK. Interglomerular center-surround inhibition shapes odorant-evoked input to the mouse olfactory bulb in vivo. $J$ Neurophysiol 95: 1881-1887, 2006.

Waldrop B, Christensen TA, Hildebrand JG. GABA-mediated synaptic inhibition of projection neurons in the antennal lobes of the sphinx moth, Manduca sexta. J Comp Physiol A 161: 23-32, 1987.

Waldrop B, Hildebrand JG. Physiology and pharmacology of acetylcholinergic responses of interneurons in the antennal lobes of the moth Manduca sexta. J Comp Physiol A 164: 433-441, 1989.

Wilson RI. Understanding the functional consequences of synaptic specialization: insight from the Drosophila antennal lobe. Curr Opin Neurobiol 21: 254-260, 2011.

Yokoi M, Mori K, Nakanishi S. Refinement of odor molecule tuning by dendrodendritic synaptic inhibition in the olfactory bulb. Proc Natl Acad Sci USA 92: 3371-3375, 1995. 